\title{
13. \\ Gleichungen der zweiten Ordnung in der Geometrie.
}

(Vom Herrn Joseph L. Raabe.)

1.

Die allgemeine Gleichung der zweiten Ordnung, zwischen zwei veränderlichen Gröfsen, ist im allgemeinen von der Form:

$$
\text { I. } A x^{2}+B y^{2}+C x y+D x+E y+1=0 \text {, }
$$

und diese Gleichung stellt eine ebene Curve vor, die auch hervorgeht, wenn eine Ebene und ein Kegel sich schneiden.

Die Grörsen $A, B, C, D, E$, nennt man die Constanten der Curve, d. h., wenn diese Gröfsen als bekannt vorausgesetzt werden, dann ist die Curve der Gröfse und Lage nach vollkommen bestimmt, und $x, y$, stellen die Coordinaten irgend eines Punctes der Curve gegen irgend ein festgesetztes rechtwinkliges Coordinatensystem vor.

Transformirt man aber das Coordinatensystem, auf welches die Gleichung (I) bezogen ist, dafs man nemlich den Ursprung der Coordinaten beibehält, und die Axe $\operatorname{der} x$ um einen Winkel a verschiebt, so dafs:

$$
\operatorname{tang} 2 \alpha=\frac{C}{A-B},
$$

dann nimmt die Gleichung (I) folgende Form an:

$$
\text { II. } A^{\prime} x^{2}+B^{\prime} y^{2}+C^{\prime} x+D^{\prime} y+1=0 \text {. }
$$

In dieser Gleichung lörmmt das Product beider Coordinaten eines Punctes nicht mehr vor. Da nun der Winkel $\alpha$ immer eine mögliche Gröfse ist, und die Gleichungen (I) und (II) dieselbe Curve vorstellen, so werden diese beiden Gleichungen nur rücksichtlich der Lage des Coordinatensystems von einander verschieden sein. Wir wollen uns daher damit beschäftigen, den Unterschied dieser beiden Gleichungen auszumitteln.

$\mathrm{Zu}$ diesem $\mathrm{Z}$ wecke wollen wir die Gleichungen der zweiten Ordnung aus dem Gesichtspuncte ableiten, dafs sie als Folgen des Durchschnittes eines Kegels mit einer Ebene angesehen werden.

2.

Es stellen $X, Y, Z$, drei sich senkrecht schneidende Coordinatenaxen vor. In der Ebene der $X Y$, befinde sich ein Punct, dessen Coor- 
dinaten, parallel mit den Axen $\operatorname{der} X$ und $Y$, durch $A$ und $B$ vorgestellt werden. In der senkrechten Distanz $m$ dieses Punctes sei der Mittelpunct eines Kreises, welcher parallel mit der Ebene der $X Y$ ist, und dessen Halbmesser $\boldsymbol{R}$ sein soll.

Ist nun der Punct, dessen Coordinaten wir durch $A$ und $B$ bezeichnet haben, der Scheitel eines Kegels, und der zuletzt erwähnte Kreis die leitende Curve, so ist die Gleichung des Kegels:

$$
(x-A)^{2}+(y-B)^{2}=\frac{R^{2}}{m^{2}} z^{2} \text {. }
$$

Nun ist $\frac{R}{m}$ die Tangente des Winkels der Seite des Kegels mit dessen Axe, und nennen wir diesen Winkel $\varphi$, dann ist die letzte Gleichung: III. $(x-A)^{2}+(y-B)^{2}=z^{2} \tan g^{2} \varphi$.

Ferner ist die Gleichung einer Ebene, die durch den Ursprung des Coordinatensystems geht, von der Form:

$$
a x+b y+c z=0 \text {. }
$$

Ist $n$ der Neigungswinkel dieser Ebene gegen die Ebene der $X Y$, und $k$ der Winkel der. Knotenlinie derselben Ebene in der Ebene der $X Y$ mit der Axe der $X$, so geht die letze Gleichung, die man hat:

$$
\frac{a}{c}=\operatorname{tang} n \sin k ; \frac{b}{c}=\operatorname{tang} n \cos k,
$$

in folgende über:

$$
\text { IV. }(x \sin k+y \cos k) \text { tang } n+z=0 \text {. }
$$

Die Gleichungen (III) und (IV) gehören der Durchschnittslinie der Ebene (IV) mit dem Kegel (III) zu. Wir wollen die Gleichung dieser Linie in der Ebene selbst, in welche sie fällt, suchen.

Denkt man sich das zuerst festgesetzte Coordinatensystem $X, Y$, $Z$, transformirt, dafs die Axe der $X$ in der alten Ebene der $X Y$ den Winkel $k$ beschreibt, wodurch die neue Axe der $x$ mit der Knotenlinie der Ebene (IV) in der Ebene der $X Y$ zusammenfällt, und sind die Coordinaten eines Punctes, dessen Coordinaten im vorigen Systeme $x, y, z$ waren, in dem jetzigen $x^{\prime}, y^{\prime}, z^{\prime}$, so hat man:

$$
\begin{aligned}
& x=x^{\prime} \cos k+y^{\prime} \sin k, \\
& y=y^{\prime} \cos k-x^{\prime} \sin k, \\
& z=z^{\prime} .
\end{aligned}
$$

Durch Substitution dieser Werthe geht die Gleichung (III) in folgende über: 
$\left(x^{\prime} \cos k+y^{\prime} \sin k-A\right)^{2}+\left(y^{\prime} \cos k-x^{\prime} \sin k-B\right)^{2}=z^{\prime 2} \tan ^{2} \varphi$, und die Gleichung (IV) nimmt folgende Gestalt an:

$$
y^{\prime} \operatorname{tang} n+z^{\prime}=0 \text {. }
$$

Es sind daher die Gleichungen der Curve gegen das so eben festgesetzte Coordinatensystem:

$$
\begin{aligned}
\left(x^{\prime} \cos k+y^{\prime} \sin k-A\right)^{2}+\left(y^{\prime} \cos k-x^{\prime} \sin k-B\right)^{2}-y^{\prime 2} \operatorname{tang}^{2} n \tan g^{2} \varphi & =0, \\
z^{\prime}+y^{\prime} \operatorname{tang} n & =0 .
\end{aligned}
$$

Dreht man ferner die Ebene der $X Y$ um die neue Axe der $x$, bis sie mit der Ebene (IV) zusammenfällt, dann sind, wenn $x^{\prime \prime}, y^{\prime \prime}, z^{\prime \prime}$ die Coordinaten eines jeden Punctes der Ebene (IV) gegen das so eben festgesetzte Coordinatensystem sind, da man hat $z^{\prime \prime}=0$ :

$$
\begin{aligned}
& x^{\prime}=x^{\prime \prime}, \\
& y^{\prime}=y^{\prime \prime} \cos n, \\
& z^{\prime}=-y^{\prime \prime} \sin n,
\end{aligned}
$$

und diese Resultate in die zwei letzten Gleichungen der Curve substituirt, erhält man die einzige Gleichung:

$\left(x^{\prime \prime} \cos k+y^{\prime \prime} \sin k \cos n-A\right)^{2}+\left(y^{\prime \prime} \cos n \cos k-x^{\prime \prime} \sin k-B\right)^{2}=y^{\prime \prime \prime} \sin ^{2} n \tan ^{2} \varphi$, für einen jeden Kegelschnitt.

Setzt man in der letzten Gleichung statt $x^{\prime \prime}, y^{\prime \prime}$, dieselben Buchstaben ohne die Zeiger, und reducirt sie gehörig, so ergiebt sich folgende Gleichung:

$$
\begin{gathered}
x^{2}+y^{2}\left(\cos ^{2} n-\sin ^{2} n \operatorname{tang}^{2} \varphi\right)-2(A \cos k-B \sin k) x \\
-2 \cos n(A \sin k+B \cos k) y+A^{2}+B^{2}=0,
\end{gathered}
$$

oder, wenn man sie durch $A^{2}+B^{2}$ dividirt, wird sie von der Form:

$$
A^{\prime} x^{2}+B^{\prime} y^{2}+C^{\prime} x+D^{\prime} y+1=0 \text {, }
$$

welche identisch mit der Gleichung (II) ist.

3.

Diejenige Gerade, welche durch den Scheitel und den Brennpunct einer Curve der zweiten Ordnung geht, nennt man $\mathrm{Hauptaxe}$ der Curve, und wenn der Schnitt des Kegels (III), welcher ein Kreis ist, die Basis des Kegels genannt wird, so fällt die Hauptaxe eines jeden Kegelschnittes mit der Geraden zusammen, welche die grölste Neigung gegen die Basis hat. Nun gehört die Gleichung (II), nach dèm so eben Gezeigten, dann einem Kegelschnitte zu, wenn das Coordinatensystem so gewählt wurde, dafs die Axe der $x$ mit der Knotenlinie der Ebene (IV) in der zuerst festgesetzten 
Coordinatenebene $X Y$ zusammenfällt. Da aber die Ebene der $X Y$ parallel mit der Basis des Kegels ist, so steht die neue Axe der $x$ senkrecht auf der Linie, welche in der Ebene (IV) liegt, und die gröfsté Neigung gegen die Basis des Kegels hat. Ist aber die Linie der grörsten Neigung zugleich Hauptaxe, oder wenigstens parallel mit der Hauptaxe der Curve, so bildet die Axe der $x$, auf welche die Gleichung (II) bezogen ist, mit der Hauptaxe der Curve einen rechten Winkel.

Verwechselt man die Axe der $x$ mit der Axe der $y$, so ist auch die Axe der $y$ auf der Hauptaxe des Kegelschnittes senkrecht. Da ferner jede auf der so eben betrachteten Hauptaxe gezogene Senkrechte parallel mit der zweiten Hauptaxe des Kegelschnittes ist, so lärst sich folgender Satz aussprechen: Wenn die Gleichung einer Curve der zweiten Ordnung von der Form (II) ist, dann liegen die Coordinatenaxen parallel mit den Hauptaxen der Curve**).

\section{4.}

Die allgemeine Gleichung der zweiten Ordnung zwischen drei veränderlichen Grörsen ist von der Form:

V. $A x^{2}+B y^{2}+C z^{2}+D x y+E x z+F y z+G x+H y+I z+1=0$.

Diese Gleichung gehört jenen Flächen an, die unter dem Namen: Flächen der zwejten Ordnung, bekannt sind. Es läfst sich aber mit den drei Coordinatenebenen, aut welche die Gleichung (V) bezogen ist, eine solche Transformation vornehmen, dafs diejenigen Glieder der letzten Gleichung, welche die Producte je zweier der Coordinaten eines Punctes der Fläche enthalten, verschwinden, wodurch dann die Gleichung (V) in folgende übergeht:

VI. $A^{\prime} x^{2}+B^{\prime} y^{2}+C^{\prime} z^{2}+D^{\prime} x+E^{\prime} y+F^{\prime} z+1=0$.

Setzt man in der Gleichung (V) $x=\alpha$, oder $y=\beta$, oder $z=\gamma$, so erhält man für einen jeden dieser Fälle, der Ordnung nach, folgende Gleichungen:

$$
\text { a. }\left\{\begin{array}{l}
B y^{2}+C z^{2}+F y z+(D \alpha+H) y+(E \alpha+I) z+A \alpha^{2}+G \alpha+1=0 \\
A x^{2}+B z^{2}+E x z+(D \beta+G) x+(F \beta+I) z+B \beta^{2}+H \beta+1=0 \\
A x^{2}+B y^{2}+D x y+(E \gamma+G) x+(F \gamma+H) y+C \gamma^{2}+I \gamma+1=0 .
\end{array}\right.
$$

*) Zwei wirkliche Hauptaxen hat nun die Ellipse; die Hyperbel und Parabel hingegen haben nur eine Hauptaxe. Man kann aber jede, auf dieser einen gezogene Senkrechte als fingirte zweite Hauptaxe ansehen. Im Kreise sind alle Durchmesser Hauptaxen, daher besteht der ausgesprochene Satz für alle Curven der zweiten Ordnung. 
Diese drei Gleichungen stellen Curven der zweiten Ordnung vor, und zwar sind diese Curven Schnitte der Fläche (V) mit Ebenen, die in den senkrechten Distanzen $a, \beta, \gamma$, von den Coordinatenebenen $y z, x z$, $x y$, angebracht sind. Da eine jede dieser Gleichungen (a) das Product der Coordinaten eines Punctes der Curven, welche sie vorstellen, enthält, so laufen daher, nach dem vorhergehenden Paragraph, die Coordinatenaxen, auf welche die Gleichung (V) bezogen ist, nicht parallel mit den Hauptaxen der Curven $(a)$.

Lälst man hingegen in der Gleichung (VI) nach und nach $x$ in $\alpha^{\prime}$, $y$ in $\beta^{\prime}$ und $z$ in $\gamma^{\prime}$ übergehen, dann stellen die so erhaltenen drei Gleichungen:

$$
\text { b. }\left\{\begin{array}{l}
B^{\prime} y^{2}+C^{\prime} z^{2}+E^{\prime} y+F^{\prime} z+A^{\prime} \alpha^{\prime 2}+1=0, \\
A^{\prime} x^{2}+C^{\prime} z^{2}+D^{\prime} x+F^{\prime} z+B^{\prime} \beta^{\prime 2}+1=0, \\
A^{\prime} x^{2}+B^{\prime} y^{2}+D^{\prime} x+E^{\prime} y+C^{\prime} \gamma^{\prime 2}+1=0,
\end{array}\right.
$$

ebenfalls die Schnitte derselben Fläche mit den drei Ebenen, die mit den Coordinatenebenen, auf welche die Gleichung (VI) bezogen ist, in den Abständen $\alpha^{\prime}, \beta^{\prime}, \gamma^{\prime}$, parallel laufen, vor.

Die Curven der Gleichungen $(b)$ sind von der Art, dafs ihre Hauptaxen parallel mit den Coordinatenaxen laufen. Man kann daher den Unterschied der Gleichurgen (V) und (VI), die derselben Fläche angehören, folgendermafsen ausdrücken: Ein jeder parallele Schnitt einer Flächéder zweiten Ordnung mit einer der Coordinatenebenen, die der Gleichung (V) zu Grunde liegen, hat seine Hauptaxen nicht parallel mit den Coordinatenaxen; während dafs ein ähnlicher Schnitt der Fläche, wo das Coordinatensystem der Gleichung(VI)zu Grunde liegt, seine Hauptaxen parallel mit den Coordinatenaxen hat.

5.

Stellt $[x, y, z]$ das senkrechte Coordinatensystem vor, auf welches die Gleichung (VI) bezogen, jede Fläche der zweiten Ordnung vorstellt, und $(x, y, z)$ jedes andere senkrechte Coordinatensystem, das denselben Anfangspunct als das vorhergehende hat, so ergiebt sich, wenn man auf die Entstehung des Coordinatensystems $[x, y, z]$ aus dem $(x, y, z)$ Rücksicht nimmt, dafs nur ein einziges Coordinatensystem von der Art möglich ist, dafs wenn man eine Fläche der zweiten Ordnung darauf bezieht, die 
Gleichung dieser Fläche jene Glieder nicht enthält, die die Producte zweier Coordinaten eines Punctes der Fläche enthalten; oder es giebt nur eine bestimmte Lage für ein senkrechtes Coordinatensystem, auf welches eine Fläche der zweiten Oudnung bezogen ist, dafs wenn man zu den Coordinatenebenén parallele Ebenen führt, diese Ebenen mit der Fläche, Curven zu Schnitten geben, deren Hauptaxen parallel mit zwei Coordinatenaxen sind.

Erinnert man sich, was unter Hauptschnitten einer Fläche der zweiten Ordnung verstanden wird, so ist klar, dafs wenn man diese drei Hauptschnitte oder je drei mit ihnen parallele Ebenen als ein Coordinatensystem ansieht, der so eben erwähnte Fall statt finden wird, nemlich: ein jeder mit einem dieser Hauptschnitte parallel geführte Schnitt zu dèr Fläche, wird auf der Oberfläche derselben eine Curve erzeugen, deren Hauptaxen parallel mit den Hauptaxen des parallelen Hauptschnittes sein werden. Da es aber nur ein einziges Coordinatensystem dieser Art geben kann, so mufs das Coordinatensystem $[x, y, z]$ parallel mit den Hauptschnitten der Fläche sein, oder: Wenn die Gleichung (VI) eine Fläche der zweiten Ordnung vorstellt, müssen die Coordinatenaxen parallel mit den Hauptaxen der Fläche sein. 